\title{
Visualization of Misfit Dislocation Network at the $\mathrm{BaSnO}_{3}-\mathrm{LaAlO}_{3}$ Interface
}

Hwanhui Yun ${ }^{1 *}$, Abhinav Prakash ${ }^{1}$, Bharat Jalan ${ }^{1}$, Jong Seok Jeong ${ }^{2}$ and K. Andre Mkhoyan ${ }^{1}$

1. Department of Chemical Engineering and Materials Science, University of Minnesota, Minneapolis, MN, USA.

2. Analytical Sciences Center, LG Chem Ltd., Daejeon, Republic of Korea.

*Corresponding author: yunxx133@umn.edu

Interface of two perovskite oxide films has been a subject of research over the past decade [1]. Broken symmetry at the interface generates characteristic atomic structures for each system, such as inherent interfacial roughness [2] or misfit dislocations originating from lattice mismatch between two crystals. In particular, misfit dislocations are routinely observed at the interfaces via cross-sectional ADF-STEM imaging [3]. The misfit dislocations are often formed as two dimensional defects at the interface. In the case of interface between two perovskite oxides with cubic crystal structure, misfit dislocation lines lie along the [100] and [010] directions. While a cross-sectional ADF-STEM image shows misfit dislocations only along the electron beam direction, a plan-view ADF-STEM image can fully visualize the entire misfit dislocation network from both directions. However, the STEM images from two layers require careful interpretation due to complicated channeling behavior of the electron beam in the materials [4]. Here, we present STEM analysis of two-dimensional (2D) misfit dislocation network at the $\mathrm{BaSnO}_{3} / \mathrm{LaAlO}_{3}$ $(\mathrm{BSO} / \mathrm{LAO})$ interface and explain the electron channeling behavior in the structure using simulations.

BSO films studied here were grown on LAO substrates by hybrid molecular beam epitaxy method [5] and plan-view TEM samples of the films were prepared by mechanical polishing. STEM images were acquired using aberration-corrected FEI Titan G2 60-300 (S)TEM operated at $200 \mathrm{keV}$. STEM image and electron intensity depth profile simulations were performed using the TEMSIM Multislice code [6].

Plan-view ADF-STEM images were obtained from $\mathrm{BSO} / \mathrm{LAO}$ heterostructures, and the patterns were studied from the image obtained in two directions. Figure 1 shows experimental and simulation focal series of ADF-STEM images using different incident beam directions ((a) BSO to LAO and (b) LAO to BSO). Observed strong dependence of ADF-STEM images on probe defocus and beam direction is explained by electron beam channeling and decaying behavior in the material. Electron channeling through two sequential layers is further explored by electron intensity depth profile simulations at different probe positions (Fig. 2). Finally, general guide to ADF-STEM imaging that enables visualization of two crystalline layers is developed, which discusses optimization of the experimental parameters for the study of buried interfaces [7].

References:

[1] HY Hwang et al., Nat. Mater. 11 (2012), p. 103.

[2] N Nakagawa, H.Y. Hwang, D.A. Muller, Nat. Mater. 5 (2006), p. 204.

[3] H Yun et al., Sci. Rep. 8 (2018), p. 10245.

[4] RJ Wu et al., Microsc. Microanal. 23 (2017), p. 794.

[5] A Prakash et al., JVSTA 33 (2015), p. 060608.

[6] AJ Kirkland in "Advanced Computing in Electron Microscopy" (Springer, New York). 
[7] This work was supported by the UMN MRSEC program (No. DMR-1420013) and a fellowship from the Samsung scholarship foundation, Republic of Korea.
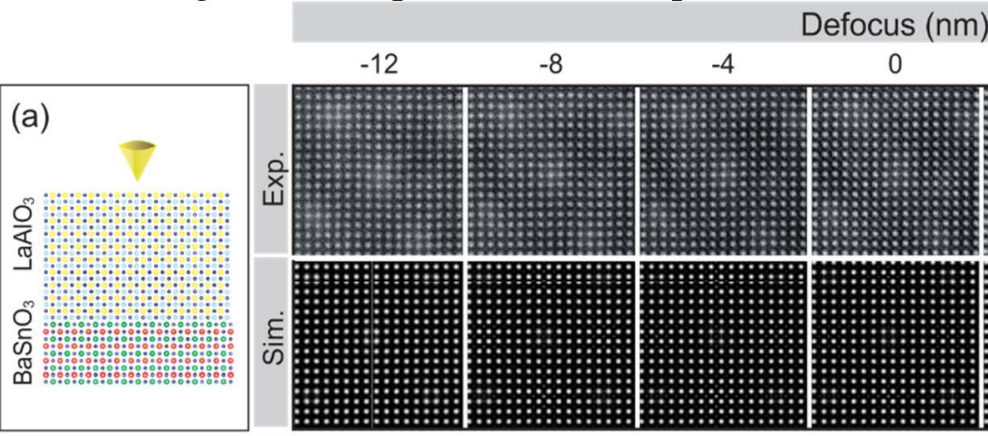

$-12$

Defocus $(\mathrm{nm})$

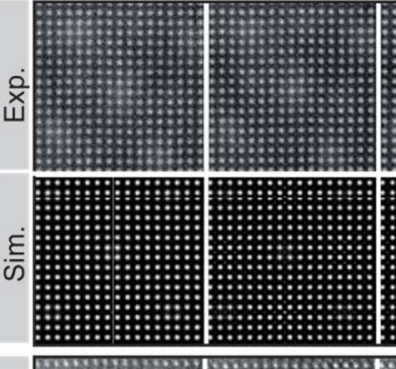

$-4$

4

8

12
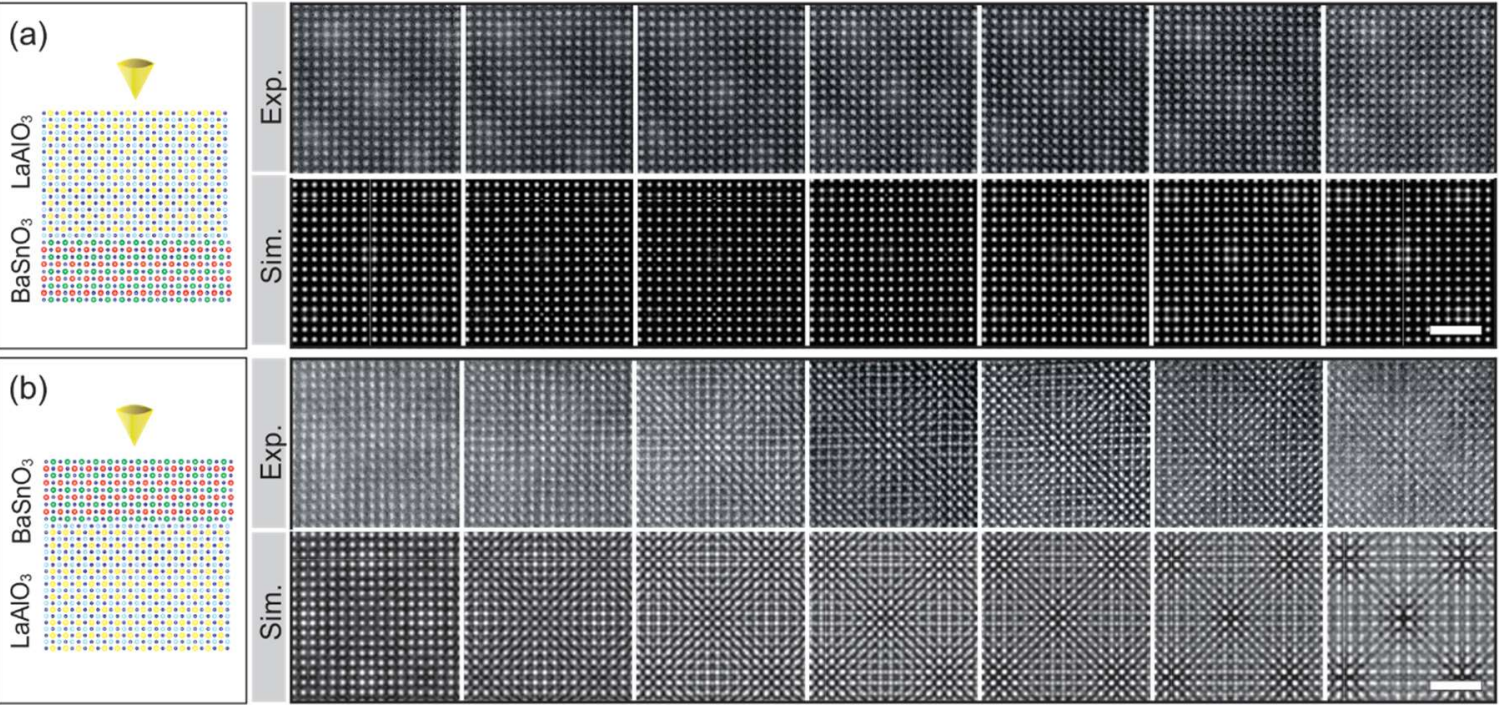

Figure 1. Experimental and simulation focal series of plan-view ADF-STEM images obtained from 10 $\mathrm{nm}-\mathrm{BSO} / 30 \mathrm{~nm}-\mathrm{LAO}$ heterostructures. Images were acquired with incident electron beam direction of (a) $\mathrm{LAO}$ to BSO and (b) BSO to LAO, respectively. Scale bars are $2 \mathrm{~nm}$.

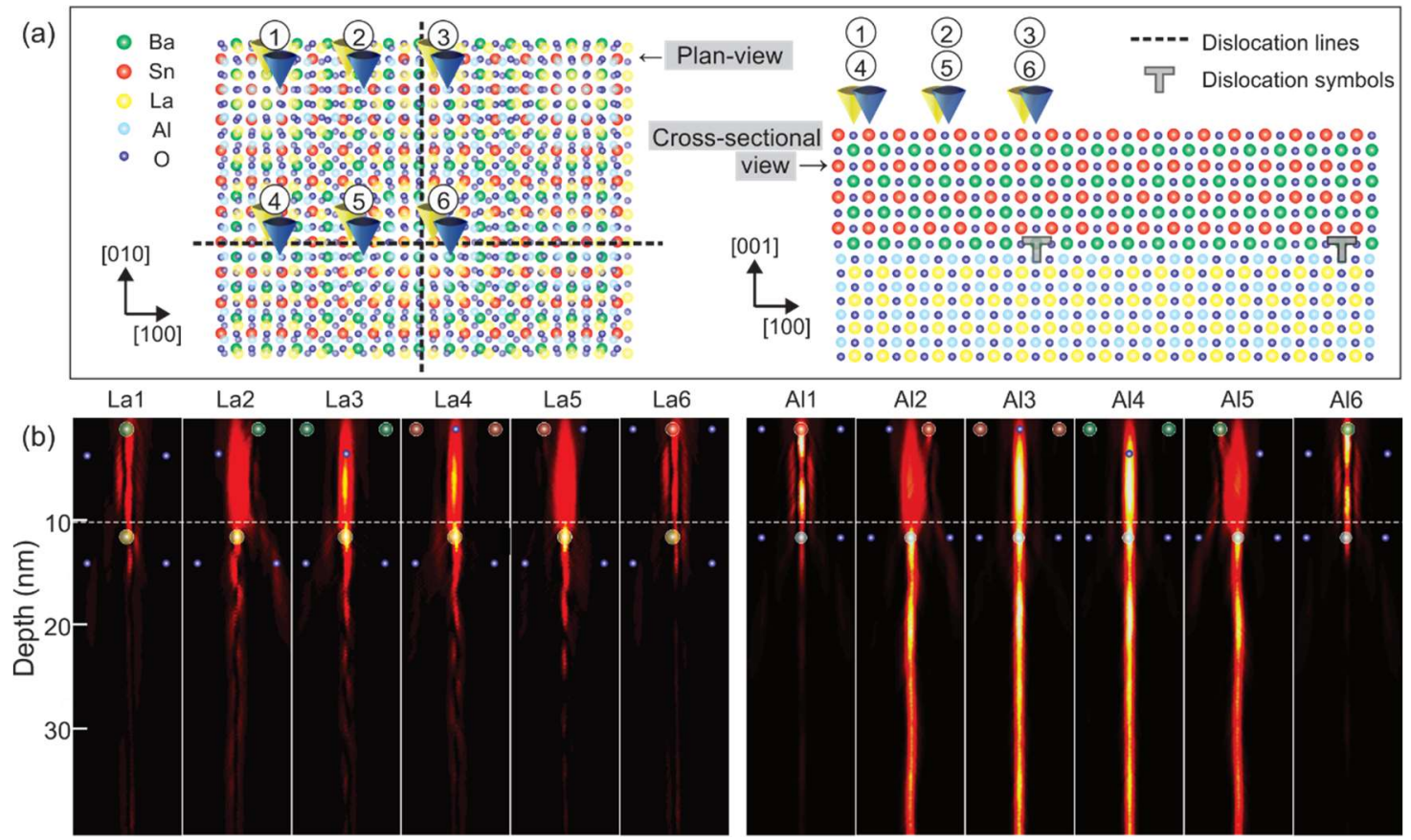

Figure 2. 2D electron intensity depth profile of a STEM probe propagating $10 \mathrm{~nm}-\mathrm{BSO} / 30 \mathrm{~nm}-\mathrm{LAO}$ heterostructures. (a) STEM probe positions chosen for the depth profile simulations are displayed on atomic models. (b) Simulated depth profiles at probe positions indicated in (a). 